\title{
Respiratory Therapist-Driven Asthma Protocols Must Be a Standard of Care
}

Among children and adolescents age $<18$ years, asthma prevalence is $>6$ million children in the United States (8.4\% of the population), resulting in almost 3.2 million asthma exacerbations annually (51\% of children with asthma experience an exacerbation). ${ }^{1}$ Both rescue and daily preventive medications are exceptionally effective today, but asthma exacerbations stem from a multitude of factors. Although the vast majority of these pediatric asthma exacerbations do not result in an emergency department visit or a hospitalization, the sheer number of annual occurrences still makes pediatric asthma one of the leading discharge diagnoses from children's hospitals in the United States for the past several decades.

Approximately 1.78 million emergency department visits $(55.9 \%$ per 10,000 of the population) result in around 189,000 (5.9\% per 10,000 of the population) annual hospitalizations for children with asthma. ${ }^{1}$ Although the number of medications for the treatment of asthma exacerbations is relatively limited, strategies for the management of acute asthma vary widely both among and within institutions. ${ }^{2}$ Diseases like asthma that have limited but scientifically validated treatment options lend themselves nicely to being managed by clinical protocols, pathways, or guidelines. Protocols are essentially operational practice guidelines for common procedures and tasks related to specific diseases. ${ }^{3}$

In 1995, McFadden et $\mathrm{al}^{4}$ found, in a sequential-design study of one of the first asthma protocols in adults, that using a protocol in the emergency department decreased hospital admissions, time spent in the emergency department, and hospital readmission. They standardized the assessment procedure, medications, administration routes, and discharge criteria. McFadden et al concluded that their protocol offered quick, efficient treatment for asthma exacerbations, substantial cost savings, and decreased unnecessary and inappropriate physician practices. ${ }^{4}$

The author has disclosed no conflicts of interest.

Correspondence: Timothy R Myers MBA RRT RRT-NPS FAARC, American Association for Respiratory Care, 9425 N. MacArthur Blvd, Suite 100, Irving, TX 75063. E-mail: timothy.myers@aarc.org.

DOI: $10.4187 /$ respcare. 07430
McDowell and associates ${ }^{5}$ published one of the few prospective controlled trials of an asthma clinical pathway for the management of status asthmaticus in children. A multidisciplinary team designed an assessment-based pathway that used a unique Algoform, combining the treatment algorithm with a form on which they recorded patient assessments and treatments. In this prospective study, an

See the Original Study on Page 1325

intensive, assessment-driven algorithm for pediatric status asthmaticus significantly reduced hospital length of stay (LOS) and costs without increasing morbidity. When asthma protocols are provided by qualified respiratory therapists, the outcomes are frequently clinically and financially positive. An RT-driven asthma protocol allows the respiratory therapist to initiate, alter, or discontinue care as the patient's condition dictates, which, ultimately, is a more cost-effective method of managing asthma. ${ }^{3}$

Myers and colleagues ${ }^{6}$ investigated the effects of an asthma care unit staffed primarily by respiratory therapists on LOS, hospital costs, charges to payers, care path variances, and postdischarge outcomes for children admitted with pediatric asthma. These investigators concluded that an asthma care unit staffed by respiratory therapists was associated with reduced LOS and care path variances, as well as lower costs of care compared to standard treatment by nurses. These outcomes were realized with no increase in postdischarge functional morbidity.

In this issue of Respiratory CARE, Miller and colleagues ${ }^{7}$ investigate the effectiveness of a pediatric asthma pathway in reducing hospital LOS for patients admitted to the pediatric ICU. The authors retrospectively reviewed the medical records of all pediatric asthma subjects 2-17 years old admitted to their pediatric ICU before ( 49 subjects) and after pathway initiation (154 subjects). The study's primary outcome was hospital LOS, and secondary outcomes were pediatric ICU LOS and time requiring continuous albuterol.

Miller et $\mathrm{al}^{7}$ showed significant decreases between the pre- and post-pathway groups in median hospital LOS $(P<.001)$, median pediatric ICU LOS $(P=.003)$, and median time on continuous albuterol $(P=.001)$. There was no significant difference between groups for pediatric 


\section{EDITORIALS}

ICU readmissions $(P=.57)$. Due to the exacerbation severity and advances in clinical practice since protocols first were instituted in the 1990s, significantly more subjects in the post-pathway group were placed on high-flow nasal cannula (HFNC) $(P=.001)$ or noninvasive ventilation (NIV) $(P=.02)$.

Miller et $\mathrm{al}^{7}$ do acknowledge these therapeutic interventions in their artice, stating that the impact of increases in HFNC and NIV on outcome measures are unclear. They also state that HFNC and NIV use were higher post-pathway despite lower heart rates and breathing frequencies, which likely reflects clinician bias toward early initiation of HFNC.

It is important to briefly discuss the limitations of this study. As with many retrospective studies, changes in clinical practice (eg, HFNC, NIV) or factors other than the pathway may have contributed to the positive outcomes. The authors also note that limitations of data available in the medical record, including a smaller pre-pathway sample size, may have also affected the outcomes. ${ }^{7}$ Additionally, the authors noted that admission heart rates and breathing frequencies were statistically significantly lower in the post-pathway group, which may have indicated that their asthma exacerbations were less severe. Based on the LOS and the actual values, however, one could make the assumption that these were relatively insignificant from a clinical standpoint. ${ }^{7}$

Asthma clinical pathways, when properly designed, carefully implemented, and closely adhered to, can result in both improved patient care and cost savings. ${ }^{2}$ While this study did not assess or determine cost of care, one could assume a decrease in cost of care between the 2 groups with a significant decrease in LOS and pediatric ICU LOS. As described by Kercsmar and Myers $^{2} 17$ years ago, improvements in patient care come in the form of reduced hospital LOS, an increased rate of clinical improvement, and decreased medication use. All these outcomes were realized in this study that investigated asthma protocols in an intensive care unit setting.

For almost three decades, asthma protocols have demonstrated positive clinical and financial outcomes for both adults and pediatric patients when respiratory therapists are given the autonomy to assess and treat. If your facility does not have an asthma protocol in place, you need to question if you are providing the evidence-based standard of care for patients with asthma.

Timothy R Myers

American Association for Respiratory Care Irving, Texas

\section{REFERENCES}

1. Center for Disease Control and Prevention. Most recent asthma data. Available at: https://www.cdc.gov/asthma/most_recent_national_ asthma_data.htm. Accessed September 25, 2019.

2. Kercsmar CM, Myers TR. Clinical pathways in treatment of asthma. Curr Opin Allergy Clin Immunol 2002;2:183-187.

3. Kallstrom TJ, Myers TR. Asthma disease management and the respiratory therapist. Respir Care 2008;53(6):770-776.

4. McFadden ER Jr, Elsanadi N, Dixon L, Takacs M, Deal EC, Boyd KK, et al. Protocol therapy for acute asthma: therapeutic benefits and cost savings. Am J Med 1995;99(6):651-661.

5. McDowell KM, Chatburn RL, Myers TR, O'Riordan MA, Kercsmar CM. A cost-saving algorithm for children hospitalized for status asthmaticus. Arch Pediatr Adolesc Med 1998;152(10):977-984.

6. Myers TR, Chatburn RL, Kercsmar CM. A pediatric asthma unit staffed by respiratory therapists demonstrates positive clinical and financial outcomes. Respir Care 1998;43(1):22-29.

7. Miller AG, Haynes KE, Gates RM, Zimmerman KO, Heath TS, Bartlett $\mathrm{KW}$, et al. A respiratory therapist-driven asthma pathway reduced hospital length of stay in the pediatric intensive care unit. Respir Care 2019;64(11):1325-1332. 